\title{
Retraction Note to: Cyber-Physical System Security Controls: A Review
}

\author{
Subhrajit Majumder, Akshay Mathur, and Ahmad Y. Javaid
}

\section{Retraction Note to:}

\section{Chapter 8 "Cyber-Physical System Security Controls:}

A Review" in: S. Guo, D. Zeng (eds.), Cyber-Physical Systems: Architecture, Security and Application, EAI/Springer Innovations in Communication and Computing, https://doi.org/10.1007/978-3-319-92564-6_8

The authors have retracted this chapter [1] because it significantly overlaps with a previously published article by Humayed et al. [2]. All authors agree to this retraction.

[1] Majumder S., Mathur A., Javaid A.Y. (2019) Cyber-Physical System Security Controls: A Review. In: Guo S., Zeng D. (eds) Cyber-Physical Systems: Architecture, Security and Application. EAI/Springer Innovations in Communication and Computing. Springer, Cham

[2] A. Humayed, J. Lin, F. Li and B. Luo, "Cyber-Physical Systems Security-A Survey," in IEEE Internet of Things Journal, vol. 4, no. 6, pp. 1802-1831, Dec. 2017. doi: 10.1109/JIOT.2017.2703172 